\title{
The Active Traveling Wave in the Cochlea
}

\author{
Thomas Duke ${ }^{1}$ and Frank Jülicher ${ }^{2,3}$ \\ 1 Cavendish Laboratory, Madingley Road, Cambridge CB3 OHE, UK \\ ${ }^{2}$ Institut Curie, Physicochimie, UMR CNRS/IC 168, \\ 26 rue d'Ulm, 75248 Paris Cedex 05, France and

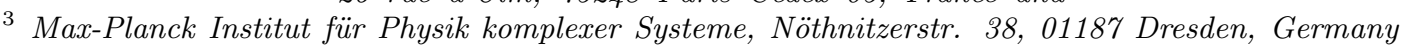

\begin{abstract}
A sound stimulus entering the inner ear excites a deformation of the basilar membrane which travels along the cochlea towards the apex. It is well established that this wave-like disturbance is amplified by an active system. Recently, it has been proposed that the active system consists of a set of self-tuned critical oscillators which automatically operate at an oscillatory instability. Here, we show how the concepts of a traveling wave and of self-tuned critical oscillators can be combined to describe the nonlinear wave in the cochlea.
\end{abstract}

PACS numbers: 87.10.+e, 47.35.+i, 05.45.-a

The sounds that we hear are detected within the cochlea of the inner ear, a fluid-filled duct which is coiled like the chamber of a snail's shell. This compartment is partitioned along almost its entire length by the basilar membrane (BM). Incoming sound waves set the BM into motion and its minute vibrations are monitored by specialized sensory hair cells [1]. The pioneering experiments of von Békésy [2], which were conducted on cadavers, demonstrated that sound excites a traveling wave on the BM, whose amplitude reaches a peak at a place which depends on the frequency. This suggested that the cochlea acts as a spatial frequency analyzer. When it became feasible to measure the BM response of living specimens, a marked difference from von Békésy's results was revealed. The sharpness of filtering was greatly enhanced and the response displayed pronounced nonlinear behavior close to resonance [3, 4, 5, 6]. These observations, together with the discovery that ears spontaneously emit sounds at specific frequencies [7], provided direct evidence for an active nonlinear amplifier in the cochlea 1], as had been foreseen by Gold [8]. But just how the ear could reliably employ an active process without suffering from unstable behavior has long been a matter of concern.

An active amplifier which overcomes this difficulty has recently been outlined $9,10,11$. It has been proposed that the cochlea contains a set of dynamical systems, each of which is maintained at the threshold of an oscillatory instability by a self-tuning mechanism. Poised at this critical point, on the verge of vibrating, each system is especially responsive to periodic stimuli at its own characteristic frequency. The concept of self-tuned critical oscillators [10] can account for the main features of hearing: sharp frequency selectivity, extreme sensitivity and wide dynamic range; and also for interference effects such as two-tone suppression and the generation of combination tones [12. In this letter, we marry the concept of critical oscillators with the physics of the traveling wave to provide a unifying description of active cochlear mechanics.

Cochlear waves.-The basic physics of cochlear waves may be described most succinctly by a one-dimensional model 13, 14, 15, 16, 17]. The BM separates the cochlear duct into two channels which are connected at the apex by a small aperture, the helicotrema. A sound stimulus impinging on the oval window, at the base of the cochlea, causes changes in the pressures $P_{1}(x, t)$ and $P_{2}(x, t)$ in both channels. Here $t$ is the time and $x$ is the position along the cochlea, with the oval window at $x=0$ and the helicotrema at $x=L$. The pressure gradients induce longitudinal currents $J_{1}(x, t)$ and $J_{2}(x, t)$, which flow in opposite directions in the two channels. We define the relative current $j \equiv J_{1}-J_{2}$ and the pressure difference $p \equiv P_{1}-P_{2}$. Then the balance of pressure gradients and inertial forces in the fluid may be written

$$
\rho \partial_{t} j=-b l \partial_{x} p,
$$

where $\rho$ is the fluid mass density, $l$ is the height of each channel, and $b$ is the breadth of the BM. The conservation of fluid volume implies that a variation in the current along the cochlea must be accommodated by a movement of the cochlear partition. We describe such deformations of the BM by its height $h(x, t)$ as a function of time and position. Then the conservation law is

$$
2 b \partial_{t} h+\partial_{x} j=0 \quad .
$$

Combining this with Eq. (11), we obtain an equation for the $\mathrm{BM}$ acceleration

$$
2 \rho b \partial_{t}^{2} h=\partial_{x}\left[b l \partial_{x} p\right] \quad .
$$

The pressure difference $p$ acts to deform the BM. If the response is passive (eg. in the dead cochlea), the response relation close to the basal end, where the stiffness $K(x)$ of the BM is high, takes the simple form

$$
p(x, t)=K(x) h(x, t) \quad,
$$

for small disturbances. Eqs. (3) \& (4) together yield a linear wave equation for the pressure, with local wave propagation velocity

$$
c(x)=\left(\frac{K(x) l}{2 \rho}\right)^{1 / 2} .
$$


Critical oscillators. - In the active cochlea, the passive response is amplified by a force-generating system. This system comprises a set of mechanical oscillators which are supported on the BM, and which are positioned in such a way that they can drive its motion. The characteristic frequency $\omega_{r}(x)$ of the oscillators is a function of position along the membrane. In general, such oscillators could either vibrate spontaneously and thus generate motion in the absence of a stimulus, or they could be quiescent and behave like a passive system. A particularly interesting case arises at the boundary of these two regimes, when every oscillator operates exactly at the critical point where it undergoes an oscillatory instability. Automatic regulation to this critical point or Hopf bifurcation - can in general be achieved by using a robust self-tuning mechanism based on local feedback control [10]. If the BM contains such self-tuned critical oscillators, its deformation $h$ in response to pressure differences across the membrane $p$ has characteristic properties as a function of frequency and amplitude, and nonlinear amplification occurs.

In order to describe this system, we first consider an individual oscillator. Its characteristic response to periodic forcing at frequency $\omega$ can be written in a general form as 10]

$$
\tilde{p}=A(\omega) \tilde{h}+B|\tilde{h}|^{2} \tilde{h} \quad .
$$

Here, $\tilde{h}$ and $\tilde{p}$ are the Fourier amplitudes at the forcing frequency and $A$ and $B$ are complex coefficients. This expression follows from a systematic expansion in the oscillation amplitude $\tilde{h}$ which is valid close to the Hopf bifurcation (comparable to a Landau expansion of the free energy of thermodynamic systems near a critical point). Proximity to an oscillatory instability thus automatically provides for generic nonlinearities. The dominant nonlinearity is cubic, a result that follows from time-translation invariance. The linear response coefficient $A$ vanishes at the characteristic frequency $\omega_{r}$ of the oscillator so that, at this particular frequency, the response becomes purely nonlinear for small amplitudes.

Thus if we focus on a particular location $x$ of the BM, its response displays a nonlinear resonance when the frequency of the stimulus is equal to the local characteristic frequency $\omega_{r}(x)$ of the oscillators. The shape of the resonance, for nearby frequencies, can be described by expanding the function $A(\omega)$ in powers of $\omega-\omega_{r}(x)$. For frequencies that differ substantially from the local characteristic frequency, on the other hand, we expect the active system to contribute little to the BM response. In particular, when $\omega=0$, the BM deflection is governed only by its passive stiffness, according to Eq. (4). We now assert that the simple functional form

$$
A(x, \omega)=\alpha\left(\omega_{r}(x)-\omega\right),
$$

where $\alpha$ is a real constant, captures the essential features of this BM response. Clearly it satisfies the requirement that the linear response coefficient at location $x$ can be expanded about $\omega_{r}(x)$. Secondly, it indicates that the passive stiffness is proportional to the characteristic frequency: $K(x)=A(x, 0)=\alpha \omega_{r}(x)$. This corresponds well with experimental data. The frequency-place map and the elasticity of the BM have been carefully measured. Characteristic frequency and stiffness both decrease approximately exponentially with distance along the cochlea, falling by about two orders of magnitude from base to apex [2, 18]. We therefore supplement Eqs. (6) \& (7) with

$$
\omega_{r}(x)=\omega_{0} e^{-x / d},
$$

to obtain the full position-dependent response of the BM. We take the coefficient $B$, describing the nonlinearity close to resonance, to be a purely imaginary constant, $B=i \beta$. This simple choice ensures that Eq. (6) has no spontaneously oscillating solution for $\tilde{p}=0$, as required at the critical point.

Active traveling waves. - Combining Eq. (3) for the BM acceleration with the response of an active membrane, described by Eq. (6), we obtain a nonlinear wave equation for the BM deformation. In frequency representation, it reads

$$
-2 \rho b \omega^{2} \tilde{h}=\partial_{x}\left[b l \partial_{x}\left(A(x, \omega) \tilde{h}+B|\tilde{h}|^{2} \tilde{h}\right)\right] .
$$

The complex solutions of this equation $\tilde{h}(x)=H(x) e^{i \phi(x)}$ describe the amplitude $H$ and the phase $\phi$ of the BM displacement elicited by a periodic stimulus with incoming sound pressure $p(x=0, t)=\tilde{p}(0) e^{i \omega t}$.

For small pressures, the nonlinearity described by the cubic term in Eq. (9) is significant only close to the resonant place which, inverting Eq. (8), is $x_{r}=d \ln \left(\omega_{0} / \omega\right)$. Far from this characteristic place, we obtain a linear wave equation which can be solved in the WKB approximation [14, 16]. The approximate solution can be expressed as

$$
\tilde{h}(x) \sim \frac{1}{\left(\omega_{r}(x)-\omega\right)^{3 / 4}} \exp \left\{i \int_{0}^{x} d x^{\prime} q\left(x^{\prime}\right)\right\},
$$

with local wave vector

$$
q(x)=\left(\frac{2 \rho}{l \alpha}\right)^{1 / 2} \frac{\omega}{\left(\omega_{r}(x)-\omega\right)^{1 / 2}} .
$$

At the basal end of the cochlea, $x<x_{r}, q$ is real and the solution is a traveling wave with a position-dependent wave vector. As the wave propagates, its wavelength diminishes and its amplitude builds up, until it approaches the place of resonance. In the immediate vicinity of the characteristic place, $A$ decreases according to Eq. (7) while $\tilde{h}$ increases. Thus the cubic term in Eq. (9) rapidly becomes more important than the linear term. This cuts off the divergence in Eq. (10) and leads to a strongly nonlinear BM response. The wave peaks at $x=x_{p}<x_{r}$, 
where the response displays the characteristic nonlinearity of critical oscillators, $\tilde{h}\left(x_{p}\right) \sim \tilde{p}\left(x_{p}\right)^{1 / 3}$ 10, 11]. From Eq. (10) we find that $\tilde{p}\left(x_{p}\right) \sim A\left(x_{p}\right)^{1 / 4} \tilde{p}(0)$, while the crossover from linear to nonlinear response implies that $A\left(x_{p}\right) \sim|B|\left|\tilde{h}\left(x_{p}\right)\right|^{2}$. We thus find that the peak amplitude has a power law response

$$
h\left(x_{p}\right) \sim p(0)^{\nu}
$$

as a function of the stimulus pressure at the base, with an exponent $\nu=0.4$. At positions beyond the characteristic place, $x>x_{r}$, the wave vector $q$ becomes imaginary, indicating the breakdown of wave propagation. The BM displacement decays very sharply in this regime.

Numerical solutions. - Full solutions to the nonlinear wave equation, Eq. (9), can be obtained numerically. It is most convenient to solve the equation for the pressure $\tilde{p}(x)$ which satisfies

$$
-\mu \tilde{p}=(A+B u(\tilde{p})) \partial_{x}^{2} \tilde{p} \quad,
$$

with $\mu=2 \rho \omega^{2} / l$ and where we have assumed for simplicity that $b$ and $l$ are constant along the cochlea. The variable $u=H^{2}$ is the squared deformation amplitude and is a nonlinear function of $\tilde{p}$. Indeed, it follows from Eq. (6) that $u(\tilde{p})$ is the unique real and positive root of the cubic equation

$$
|\tilde{p}|^{2}=|A|^{2} u+\left(A^{*} B+A B^{*}\right) u^{2}+|B|^{2} u^{3} .
$$

Eq. (13) for $\tilde{p}$ can be solved, starting from $x=L$ and integrating towards $x=0$. As a boundary condition, we impose zero pressure difference at the helicotrema, $\tilde{p}(L)=0$, because the two cochlear channels are connected there. A second boundary condition specifies the value of $\partial_{x} \tilde{p}$ at $x=L$. By varying this pressure gradient at the helicotrema, we find solutions that correspond to waves entering the cochlea at $x=0$ with different pressure amplitudes $\tilde{p}(0)$. The profile of $\mathrm{BM}$ displacements can then be obtained from the solution $\tilde{p}(x)$ via

$$
\tilde{h}=\frac{\tilde{p}}{A+B u(\tilde{p})} \text {. }
$$

Basilar membrane response.-Examples of traveling waves are displayed in Fig. 1 for two different sound levels and varying stimulus frequencies. Waves initiated at $x=0$ propagate with growing amplitude and decreasing wavelength until they reach a point of resonance, beyond which they decay rapidly. At $40 \mathrm{~dB}$ SPL, the resonance is sharp and the peak response occurs at a location very close to the characteristic place $x=x_{r}$, where the frequency of the active oscillators is equal to the stimulus frequency. At $80 \mathrm{~dB}$ SPL, the resonance is much broader and the location $x=x_{p}$ of maximal response shifts towards the base, in agreement with experimental observations [6].

The response at a particular location on the BM exhibits the qualitative properties of cochlear tuning that
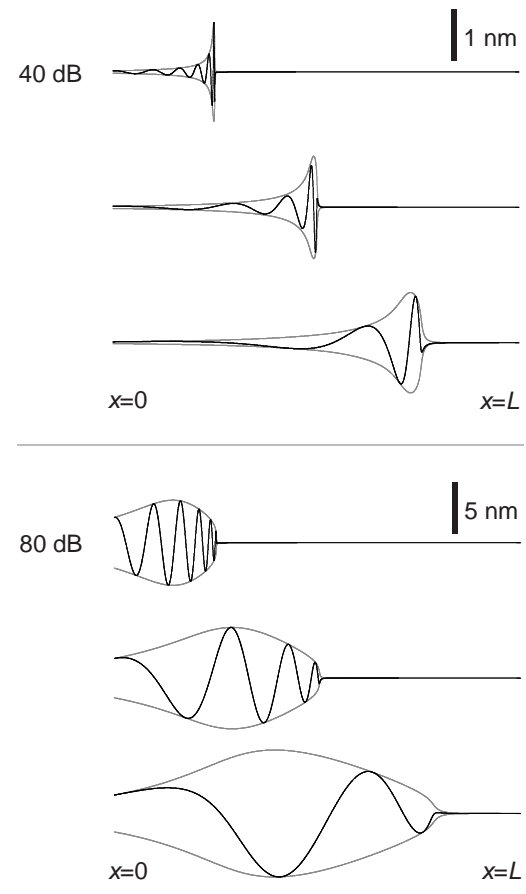

FIG. 1: The active traveling wave on the BM for three different frequencies $(f=370 \mathrm{~Hz}, 1.3 \mathrm{kHz} \& 4.6 \mathrm{kHz}$ ) whose corresponding characteristic places are $x_{r} / L=0.25$ (top), 0.5 (center) \& 0.75 (bottom). Instantaneous BM displacement $h(x, t)$ (black lines) and wave amplitude $H(x)$ (gray lines) are shown for two stimulus amplitudes, characterized by sound pressure level (SPL). At $40 \mathrm{~dB}$ SPL, a sharp resonance occurs at the characteristic place, where the critical oscillators actively drive the response. The resonance is broader at $80 \mathrm{~dB}$ SPL, and the peak shifts towards the base of the cochlea. Parameter values: Cochlear dimensions $L=35 \mathrm{~mm}$ and $l=1 \mathrm{~mm}$; fluid density $\rho=10^{3} \mathrm{~kg} / \mathrm{m}^{3}$; parameters governing the frequencies of the active oscillators $\omega_{0}=10^{5} \mathrm{~s}^{-1}$ and $d=7 \mathrm{~mm}$, providing a frequency range of $100 \mathrm{~Hz}-16 \mathrm{kHz}$. We choose $\alpha=10^{4} \mathrm{~Pa} / \mathrm{ms}$, which implies a volumetric stiffness of the $\mathrm{BM}$ varying in the range $6 \times 10^{6}-10^{9} \mathrm{~Pa} / \mathrm{m}$. There is only one free parameter in our calculations, $\beta=10^{23} \mathrm{~Pa} / \mathrm{m}^{3}$, which we choose to fit the nonlinearity of the response according to sound pressure level (SPL). For simplicity, it is assumed that the middle ear raises sound pressures by $20 \mathrm{~dB}$, independent of frequency.

have been observed experimentally $3,4,5]$. Fig. 2a displays the gain $\omega|\tilde{h}| /|\tilde{p}(0)|$ of BM velocity, obtained from our numerical solutions, as a function of stimulus frequency for different sound levels. At low frequencies the response is linear and the gain is independent of the stimulus amplitude. As the stimulus frequency approaches the resonant frequency, the response becomes nonlinear and the gain diverges as the SPL declines. At higher frequencies, the response drops precipitously. The magnitude of the BM displacement, shown in Fig. 2b, is typically several nanometers at resonance, in quantitative agreement with experimental data [4]. The phase $\phi$ of the traveling wave at a particular location on the BM is 

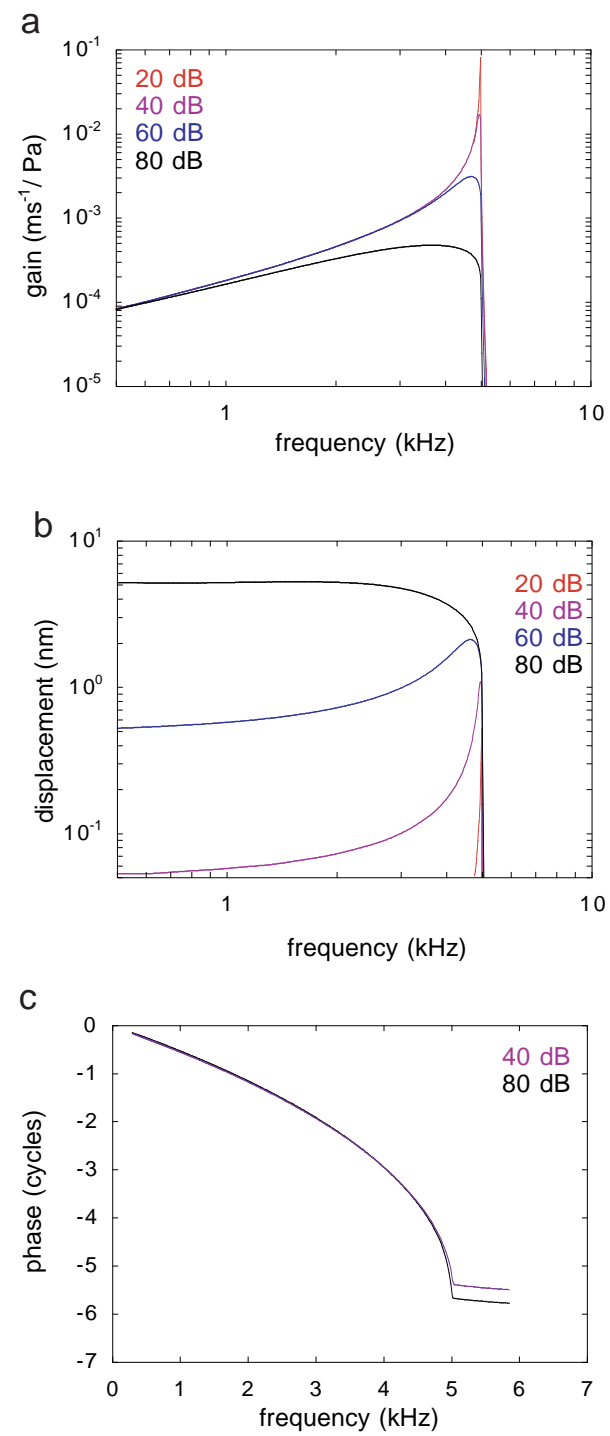

FIG. 2: Response of the BM at a fixed position as a function of stimulus frequency, for different sound pressures. The characteristic frequency of the active oscillators at that location is $5 \mathrm{kHz}$. (a) Velocity gain, i.e. BM velocity divided by sound pressure of the stimulus. (b) Corresponding BM displacement. (c) Phase difference between stimulus and BM oscillation.

displayed in Fig. 2c. As observed experimentally, it decreases with increasing frequency - gradually at first, but more abruptly as resonance is approached - and then varies only little at frequencies higher than the characteristic frequency.

Discussion.-In many recent cochlear models, sections of the BM are considered to behave as inertial oscillators which are either lightly damped (in passive models) 14, 15, 16 or driven by internal forces (in active models) 19, 20, 21]. The characteristic frequency at a particular location is then the local resonant frequency of the BM, which varies as the square root of the stiffness. A prob- lem with this interpretation is that, in order to obtain the observed range of characteristic frequencies, the stiffness of the BM would have to vary by more than four orders of magnitude from base to apex. The measured variation is only a factor of one hundred 2, 22]. This difficulty is circumvented by our theory, where the range of frequencies at which the BM resonates is determined by the frequencies of the oscillators that are ranged along it, and is not governed by the stiffness or the inertia. Some models of the active cochlea are very specific and rely on additional degrees of freedom, secondary resonances or time-delayed feedbacks 21]. Such descriptions lack the simplicity and generality of our approach and miss the generic nature of the power-law nonlinearities [10, 11, 23] conferred by the Hopf bifurcation.

In this letter it has been our aim to provide a concise, coherent interpretation of a wide variety of observations, rather than a detailed fit of individual data. Considering that our model incorporates only one free parameter whose value is not determined by independent measurement, the qualitative agreement with a diverse set of experimental data is striking. We have not sought to specify the physical nature of the active oscillators. The electromotility of outer hair cells has been implicated in active amplification in the mammalian cochlea [1, 24, 25], but the motile response of hair bundles may also play a role. Indeed, the hair bundles of frog hair cells have recently been demonstrated to behave as Hopf oscillators [26, 27]. Because the response of self-tuned critical oscillators is generic, our analysis remains valid whatever the physical basis of force generation.

We thank M. Magnasco, P. Martin, E. Olson, J. Prost and G. Zweig for stimulating discussions. T.D. is a Royal Society University Research Fellow.

[1] P. Dallos, A.N. Popper \& R.R. Fay (Eds.), The Cochlea (Springer, New York 1996).

[2] G. von Békésy, Experiments in Hearing (McGraw Hill, New York 1960).

[3] W.S. Rhode, J. Acoust. Soc. Am. 49, 1218 (1971).

[4] M.A. Ruggero et al., J. Acoust. Soc. Am. 101, 2151 (1997).

[5] L. Robles \& M.A. Ruggero Physiol. Rev. 81, 1305 (2001).

[6] I.J. Russel \& K.E. Nilsen, Proc. Natl. Acad. Sci. USA 94, 2660 (1997).

[7] D.T. Kemp, J. Arch. Otorhinolaryngol. 224, 37 (1979).

[8] T. Gold, Proc. Roy. Soc. B 135, 492 (1948).

[9] Y. Choe, M.O. Magnasco \& A.J. Hudspeth, Proc. Natl. Acad. Sci. USA 95, 15321 (1998).

[10] S. Camalet, T. Duke, F. Jülicher \& J. Prost, Proc. Natl. Acad. Sci. (USA) 97, 3183 (2000).

[11] V.M. Eguiluz et. al., Phys. Rev. Lett. 84, 5232 (2000).

[12] F. Jülicher, D. Andor \& T. Duke, Proc. Natl. Acad. Sci. USA 98, 9080 (2001).

[13] J. Zwislocki, Acta Otolaryngol. suppl. 72 (1948).

[14] G. Zweig, Cold Spring Harbor Symp. Quant. Biol. 40, 
619 (1976)

[15] E. de Boer, Phys. Rep. 62, 87 (1980).

[16] J. Lighthill, J. Fluid. Mech. 106, 149 (1981).

[17] G. Zweig, J. Acoust. Soc. Am. 89, 1229 (1991).

[18] D.D. Greenwood, J. Acoust. Soc. Am. 87, 2592 (1990).

[19] H. Duifuis et.al., in Peripheral Auditory Mechanisms, edited by J.B. Allen et. al. (Springer, Berlin 1985).

[20] P.J. Kolston, E. de Boer, M.A. Viergever \& G.F. Smoorenburg J. Acoust. Soc. Am. 88, 1794 (1990).

[21] E. de Boer, chap. 5 in Ref. 1 \& references therein.

[22] R.C. Naidu \& D.C. Mountain, Hearing Res. 124, 124
(1998).

[23] M.O. Magnasco, Phys. Rev. Lett. 90, 058101, (2003).

[24] W.E. Brownell, C.R. Bader, D. Bertrand \& Y. de Ribaupierre, Science 227, 194 (1985).

[25] J. Ashmore, J. Physiol. 388, 323 (1987).

[26] P. Martin, A.J. Hudspeth \& F. Jülicher, Proc. Natl. Acad. Sci. 98, 14380 (2001).

[27] P. Martin \& A.J. Hudspeth, Proc. Natl. Acad. Sci. 98, 14386 (2001). 